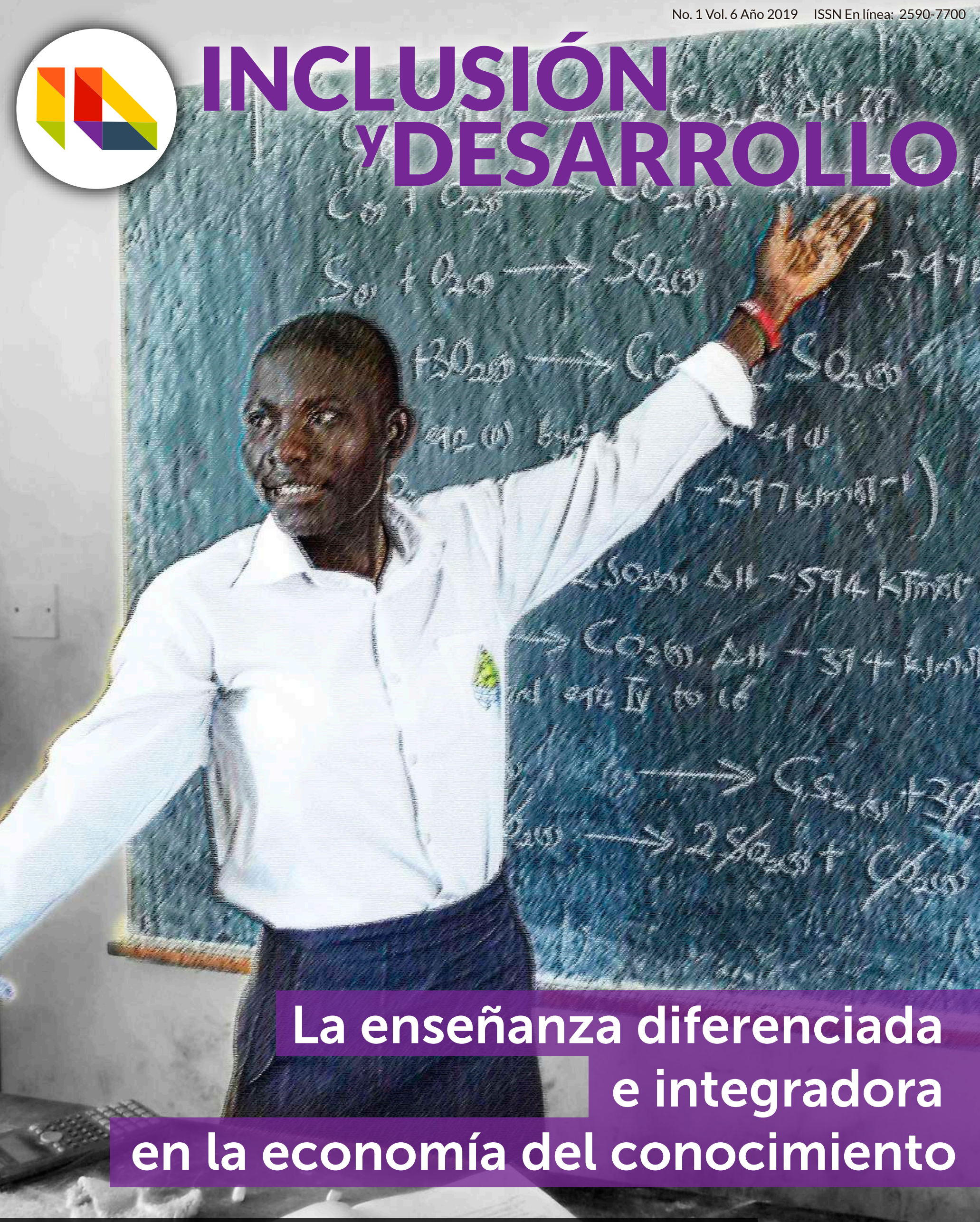

Marta Mercedes Fernández Guerrero y Clara Inés Sagre Hernández 


\section{LA ENSEÑANZA DIFERENCIADA E INTEGRADORA EN LA ECONOMÍA DEL CONOCIMIENTO}

\section{THE DIFFERENTIATED AND INTEGRATING TEACHING IN THE KNOWLEDGE ECONOMY}

\author{
Marta Mercedes Fernández Guerrero \\ marta.fernandez.19@hotmail.com \\ Fundación Tecnológica Antonio De Arévalo -TECNAR \\ Cartagena, Colombia
}

Clara Inés Sagre Hernández clara.sagre@tecnar.edu.co Fundación Tecnológica Antonio De Arévalo -TECNAR

Cartagena, Colombia

\section{Resumen}

Las reflexiones que se describen en este artículo se fundamentan en el principio que la educación es el factor más influyente en el progreso equitativo de los pueblos, lo cual exige que la enseñanza que se imparte induzca a la persona a un aprendizaje que genere habilidades y destrezas basado en principios y valores, para que comprenda y aplique el conocimiento en correspondencia con las exigencias del mercado laboral de tal forma que pueda contribuir al desarrollo socioeconómico de esta sociedad, que hoy también se conoce como Economía del Conocimiento, en donde el Ser Humano participa como actor del proceso productivo y también se convierte en el artífice y autor de nuevas tendencias y procesos que resultan de ese conocimiento que surge de la investigación, de la exploración constante y que le permite aplicarlo e innovar y así poder acrecentar la ciencia y la técnica que requiere el contexto en que se vive, contribuyendo a disminuir la inequidad al generar nuevas alternativas en el mercado laboral. En consecuencia, la educación debe ser apreciada y aplicada como un proceso continuo que les permita a las personas estar acordes con la dinámica de la ciencia y la técnica y así asegurar una estabilidad laboral a través de su vida; por lo tanto, los sistemas educativos de los países deben armonizar con esta dinámica. En el caso colombiano, la estructura del sistema educativo, en el nivel superior, permite una formación secuencial o por ciclos propedéuticos exigiendo una enseñanza diferenciada e integradora que genere en el estudiante habilidades y destrezas coherentes con los propósitos de cada nivel de formación.

Palabras clave: educación, profesor, estudiante, formación secuencial, enseñanza diferenciada e integradora, economía del conocimiento. 


\section{Abstract}

The reflections that are described in this article are based on the principle that education is the most influential factor in the equitable progress of the people; which demands teaching to introduce the individual in a learning way that generates abilities and skills based in principles and values, in order to understand and practice knowledge, based on the demands of the labor market, so as to contribute to the development of this society, nowadays it is known as Knowledge Economy where the human being has a role on the productive process becoming in architect and author as well, of new trends that result from that knowledge that arises from research and constant exploration, allowing it to apply and innovate and thus to be able to increase the science and technique required by the context in which it lives, helping to reduce inequality generating new alternatives in the labor market. Consequently, education must be appreciated and applied as a continuing process which allows people to go along with science and technique dynamics, in order to assure working stability through their lives; therefore, national education systems must be coherent with this dynamic. In Colombia, the higher education level, the structure of the educational system allows a sequential training, demanding a differentiated and integrating teaching that engenders in the student abilities and skills consistent with the purposes of each level of training.

Keywords: education, teacher, student, sequential training, differentiated and integrating teaching, knowledge economy. 


\section{INTRODUCCIÓN}

a vida del ser humano siempre ha estado Lasociada a la búsqueda de la verdad. Su comportamiento demuestra que posee una sabiduría innata que ha incidido en el ordenamiento de todas las variables que determinan su diario vivir. Sin embargo, hoy en día, gracias a la educación y a la accesibilidad a la misma, esa sabiduría está mejor estructurada permitiéndole a las personas aprovechar y acrecentar la Ciencia y la Técnica, así como controlar su instinto prevaleciendo una razón equilibrada que puede propiciar mejores ambientes de convivencia.

El reconocimiento explícito que hoy se tiene del ser humano, como impulsor de los adelantos científicos y técnicos, generadores de tecnologías, influyó para que este siglo XXI, desde sus inicios, fuera conocido como la Sociedad del Conocimiento, y la incidencia de estos adelantos en los procesos productivos determinó que, igualmente, se referencie con la expresión Economía del Conocimiento. Dos locuciones interrelacionadas, ya que un conocimiento ordenado, dinámico en el tiempo y responsable genera ventajas competitivas con el fin de asegurar la efectividad de la economía de un País.

Estos adelantos científicos y técnicos han generado cambios muy significativos en los ámbitos socioeconómico y geopolítico, los que se vienen observando principalmente en las últimas décadas del siglo pasado y en los albores del presente y que han promovido una nueva forma de establecer relaciones económicas y sociales entre los países, que trascienden las fronteras y genera cambios en el mercado laboral.

Así las cosas, es necesario que la fuerza laboral adquiera una formación pertinente con la dinámica de la Ciencia y la Técnica y la aplicación de tecnologías producto de estos desarrollos, exigiéndoles a las personas una disposición hacia la formación permanente; es decir, una predisposición a "aprender para la vida" y por consiguiente esta forma de apreciar la incidencia de los adelantos científicos y tecnológicos, demandan cambios estructurales en el sistema educativo, en la forma de enseñar, para que la fuerza laboral responda a las nuevas exigencias del entorno.

Ante esta evolución sistemática de la sociedad actual, se afianza la concepción de que la educa- ción es una estrategia intrínseca y determinante para avanzar hacia el desarrollo social integral basado en la distribución equitativa de la riqueza, lo que requiere de personas con sabiduría espiritual e intelectual resultado de un saber interdisciplinario apoyado en un saber hacer pertinente y sustentando en un saber ser que humanice la apropiación y aplicación de la ciencia y la técnica. El sistema educativo debe ajustarse a estas realidades, en consecuencia, la administración de la educación debe estar soportada en procesos dinámicos y flexibles enmarcados en la internacionalización generada por el desarrollo de las comunicaciones, lo que demanda que los agentes que intervienen en la gestión académica también posean una formación acorde con este escenario.

Estas reflexiones conllevan a enfatizar en la formación del profesor como agente determinante de una enseñanza secuencial que propicie un aprendizaje gradual, coherente con los intereses personales, sociales, económicos y de familia, generando un aprendizaje para la vida que le permita a la persona desarrollar, en el tiempo, su formación; que no pierda nada de lo aprendido, que se materialice y aproveche en el saber y en el hacer.

\section{Desarrollo}

La educación es un medio eficaz para adaptarse a las nuevas exigencias del entorno y para ello debe propiciar la formación de personas capaces de insertarse en el mercado laboral y aprovechar las ventajas que resultan de la interacción de la ciencia y la técnica. Al respecto, Gómez, B. H., manifiesta que "En la sociedad del conocimiento el valor agregado ya no proviene de los factores clásicos de la producción 'tierra, capital y trabajo': viene de la tecnología antes que nada" (1999, p. 3), Afirmación que reconoce a las ventajas competitivas como dinamizadoras de las relaciones socio-económicas, las que son producto de la creatividad que genera el conocimiento humano, dejando en el pasado la concepción de las ventajas comparativas propias de la naturaleza y las que en su momento se apreciaban como las impulsoras del crecimiento de los pueblos.

El apreciar a las ventajas competitivas como eje central de una economía del conocimiento no significa que se relegan las ventajas comparativas o naturales, ellas continúan siendo la base 
del desarrollo socioeconómico de los países pero ahora, el ser humano con un conocimiento bien estructurado, generará un mayor valor agregado a estas ventajas naturales. En este orden de ideas Ferranti, Perry, Lederman y Maloney (2002) manifiestan que para aprovechar mejor los recursos naturales es necesario fortalecer los siguientes aspectos:

- Educación general y aprendizaje durante toda la vida

- Incentivos en materia de Investigación y Desarrollo, y mecanismos de innovación

- La función especial de la Tecnología de la información y las comunicaciones

- Infraestructura pública,

- Buenas Instituciones

Y con relación a la educación, afirman:

Las medidas encaminadas a crear sistemas de educación que susciten una educación general de alta calidad, pero además centrada en el aprendizaje y la capacitación durante toda la vida, son fundamentales para construir el capital humano (...). Esto también es un requisito previo para la instauración de fuertes mecanismos para estimular la innovación. (p. 4).

Estas nuevas características que se vienen observando no pueden ser ignoradas por el sector educativo, por ello es necesario tener claridad de lo que se pretende en la llamada Economía del Conocimiento para ajustar los procesos de enseñanza-aprendizaje a estas exigencias y entregarle a la sociedad el recurso humano que se requiere. $\mathrm{Al}$ respecto Saldaña y Barragán dicen:

Una economía basada en el conocimiento, hace referencia a un modelo de desarrollo en el cual el conocimiento (desarrollo científico y tecnológico, habilidades, entrenamiento profesional) es un factor primordial de producción de bienes y servicios de alto valor agregado, competitivos a nivel internacional. El conocimiento y la innovación se convierten en elementos fundamentales para la creación de riqueza (2016, p. 1).

Sánchez y Ríos (2011) manifiestan que la economía del conocimiento "es aquella en la que el conocimiento es un activo más importante que los bienes de capital y mano de obra, y donde la cantidad y sofisticación del conocimiento que permea en las actividades económicas y sociales, llega a niveles muy altos" (p. 46); o sea, se requiere de personas con habilidades que induzcan su saber hacia la innovación creando un valor agregado que fortalezca la economía incidiendo en un mejor vivir de la población al superar esa inequidad que prevalece en la mayoría de los países del mundo y Colombia es uno de ellos.

Por esta razón, es de imperiosa necesidad que la sociedad en general, especialmente las comunidades académicas, reflexionen sobre las singularidades de la Educación, en particular, la forma cómo debe darse la enseñanza con el propósito de que el aprendizaje sea pertinente con la actualidad. La acción de enseñar ha sido expresada, a través de la historia, de diferentes formas, pero todas ellas apuntan a una concepción fundamental: La enseñanza es la que determina el crecimiento intelectual y espiritual del ser humano en pro de una vida más agradable y digna. La enseñanza se constituye en un elemento neurálgico para asegurar la buena calidad ${ }^{1}$ de la educación. Por consiguiente, es relevante preguntarse: ¿Qué características debe tener una enseñanza -y quien la orienta- para formar las personas que requiere esta sociedad llamada Economía del Conocimiento?

Al tratar de dar respuesta a este interrogante nos suscribimos al sector de la educación superior teniendo en cuenta las singularidades del Sistema Educativo Colombiano y, acogiendo aspectos teóricos para identificar con objetividad las características de un proceso de enseñanza que forme a las personas que requiere esta sociedad, en donde el Ser Humano es el eje de todo actuar que conduzca al bienestar de su entorno, con la incidencia propia de las relaciones socioeconómicas y geopolíticas de este mundo globalizado.

\section{REFERENTES CONCEPTUALES}

El Estado debe concientizarse que la educación es el principal factor para que un país optimice el uso de sus recursos a partir de una Economía basada en el Conocimiento, con el fin 
de lograr que el pensar de la Nación se enfoque hacia el aprovechamiento objetivo y responsable de la riqueza, lo que es posible cuando las personas desarrollan capacidades y actitudes que le permiten intervenir con efectividad en el mercado laboral. Al respecto, el Banco Mundial en su publicación Construir Sociedades de Conocimiento: Nuevos Desafíos para la educación terciaria. (2002) expresa que:

El estado tiene la responsabilidad de establecer un marco favorable que impulse a las instituciones de educación terciaria a ser más innovadoras y a responder mejor a las necesidades de una economía del conocimiento globalmente competitiva, así como a las nuevas exigencias del mercado laboral en términos de capital avanzado (p. xx).

En un aparte de la Declaración Universal de los Derechos Humanos (1948), citado en la Guía para la Internacionalización elaborado por el Instituto Colombiano para el Fomento de la Educación Superior-ICFES, dice que: "toda persona tiene derecho a la educación"...y "el acceso a los estudios superiores será igual para todos, en función de los méritos respectivos" (2002, p. 215). Apreciación que cada día es aceptada e implementada con mayor objetividad por la incidencia de la educación en el desarrollo de los pueblos.

La educación superior, asociada con la educación terciaria ${ }^{2}$, juega un papel determinante en la Economía del Conocimiento y por consiguiente es relevante para la consolidación de la democracia de una Nación, ya que propicia, como se viene manifestando, un crecimiento económico que debe ser equitativo y continuo para generar bienestar en la población.

Las reflexiones expuestas, exigen una actitud de cambio en los implicados directos del sector educativo: Estado, Instituciones de Educación, Profesor y Estudiante. Esta actitud debe basarse en el trabajo en equipo que conciba el conocimiento y la técnica como una realidad determinante de la Sociedad del Conocimiento.
En este orden de ideas, el Centro Interuniversitario de Desarrollo - CINDA, en su documento Aseguramiento de la Calidad en Iberoamérica. Educación Superior, manifiesta que:

La expansión de los sistemas de educación superior, la diversificación de la oferta y la mayor heterogeneidad del cuerpo de estudiantes han demostrado la necesidad de abrir el campo y dejar la visión tradicional de la educación superior para visualizar una más amplia, <que llaman educación terciaria> (2012, p. 24).

En cuanto a la población heterogénea, "La expansión del cuerpo estudiantil no sólo significa mayor número sino mayor diversidad: edad, género, calificaciones, capital cultural y expectativas" (CINDA, p. 25). Característica que se convierte en un desafío para el sector educativo que tiene el compromiso social de preservar la calidad de la educación. Por su parte, la Agenda 2030 para el Desarrollo Sostenible, denominada Transformando Nuestro Mundo, y definida en el 2015 con participación de líderes mundiales, identificó 17 propósitos como los Objetivos del Desarrollo del Milenio (ODM) teniendo como eje central a las personas y al mejoramiento del planeta, y una de las metas es disminuir la pobreza, lo que lógicamente contribuye a disminuir la desigualdad entre las personas (Martínez A., M., 2015).

Lograr los ODM de la Agenda 2030 reafirma el compromiso que tiene el sector educativo -así como los agentes intervinientes- en su gestión con la sociedad en lo relacionado con la formación de personas capaces de desempeñarse adecuadamente en el mercado laboral al incidir en el mejor aprovechamiento de los recursos.

Con relación a las Instituciones de Educación Superior - IES, deben reestructurar su gestión académico-administrativa y orientarla a las nuevas exigencias del mundo globalizado, por lo que deberán contar con personal bien preparado que identifique los programas académicos pertinentes a la realidad del entorno socio-económico, un cuerpo docente con una formación integral (disciplinar, pedagógica, con actitudes éticas) y 
estar regentadas por directivos conscientes de la responsabilidad que les embarga como administradores de la educación.

En este sentido, el Profesor es quien incide en forma directa en todas las pretensiones de mejoramiento de la educación por ser el orientador del proceso de enseñanza - aprendizaje y el agente que propicia un aprendizaje responsable. Su participación reflexiva, crítica y efectiva favorece "a la innovación, la renovación, la integración de la diversidad y el rendimiento de los alumnos" (Macías, 2001, p. 611).

En la educación superior el profesor, centro de atención de estas reflexiones, "es el profesional de la docencia que muestra en su actividad cotidiana -docencia e investigación- conocimiento, interés y compromiso, además de aplicar métodos pedagógicos que hacen de la enseñanza y la investigación una realidad inseparable" (González y Macías, 2004, p. 303).

Desde esta concepción, Borrero Cabal, S.J., expresa que:

De cada profesor -o mejor, de cada maestrose espera que sepa vivir en estado de sobresalto intelectual, de redundancias y resultancias de su espíritu inquisitivo en torno a la ciencia de su dominio y de su acertado dominio pedagógico, porque a su disciplina él la ama de corazón, la vive al día y le busca caminos decentes, sin envejecimientos y exposiciones rutinarias (2001, p. 6).

Estas características del docente que expone Borrero, se complementan con el hecho de que "los profesionales de la educación tienen sobre sí un intenso y extenso trabajo de lo cual algunos no son conscientes y otros tratan de ocultarlo donde el propio sistema se lo permite y propicia" (González, 2005, p. 715). Ambas actitudes son superables cuando el ejercicio de la enseñanza está acompañado de estímulos que la posicionen como una actividad laboral que les mejora su bienestar socioeconómico, lo cual contribuye a atenuar presiones que les dispensen su desempeño al tener satisfechas las necesidades básicas de su familia y las propias.

El Estudiante es el beneficiario directo de todo proceso de enseñanza-aprendizaje, lo que requiere considerar las singularidades que lo dis- tinguen en este entorno en el que se visiona como un ciudadano del mundo, en ejercicio del pleno desarrollo de su personalidad; respetuoso de los derechos, deberes y la diversidad cultural, étnica y ambiental; en paz y armonía con sus semejantes y la naturaleza; con capacidad para acceder al conocimiento científico, técnico, cultural y artístico; un ser autónomo y competente en su desempeño personal, social y laboral (Fernández, G. M, 2008, p. 26).

\section{RefERENTES CONTEXTUALES}

\section{Generalidades}

La importancia que una nación le da a la educación se refleja en el interés de los gobiernos, de las organizaciones nacionales e internacionales, de las mismas instituciones y de su comunidad académica, expresándolas en sus planes de desarrollo, resultados del debate sobre las tendencias teóricas y prácticas de los procesos educativos y su coherencia con los requerimientos del contexto; debates interactivos en donde deben participar no sólo el sector educativo sino también el empresarial, la sociedad civil y el Estado, en procura de buscar respuestas coordinadas y pertinentes.

Estas tendencias se observan en la mayoría de los países pero, a pesar del reconocimiento que se le hace a la educación como estrategia de desarrollo socioeconómico, hay regiones en el contexto mundial que, aun cuando son conscientes de su importancia, no están implementando acciones para darle el posicionamiento que amerita por no contar con una actitud contundente al respecto, especialmente en lo referente a los elementos determinantes como es el caso de la asignación del presupuesto que se requiere para lograr ese reconocimiento.

\section{Particularidades (caso Colombia)}

En Colombia, el Gobierno se está esforzando por posicionar la educación como estrategia dinamizadora del desarrollo económico, tal como se refleja en el programa Visión Colombia II Centenario - 2019, en el que se resalta la importancia de la formación básica obligatoria y además se destacan la formación Técnica Profesional y Tecnológica como vectores para apoyar el fortalecimiento de procesos productivos y de servicios en el país. Inclusive, en estos momentos, se está 
revisando su estructura, armonizándola a la concepción que se viene aplicando en algunos países y conocida como educación terciaria.

Hoy, la educación superior en Colombia es regida por las Ley 30 de 1992 y en su Artículo 1 la define como "un proceso permanente que posibilita el desarrollo de las potencialidades del ser humano de una manera integral". Igualmente, en esta misma Ley, se reglamenta el nivel de pregrado en forma secuencial o por ciclos propedéuticos: técnica profesional, tecnológica y profesional universitaria, así como lo concerniente al aseguramiento de su calidad.

Por su parte, en la Ley 749 del 19 de julio del 2002 se identifican las competencias laborales propias de los niveles enunciados. Esta estructuración secuencial le permite a la persona formarse de manera gradual de acuerdo con sus potenciales intelectuales, su interés personal, disponibilidad de tiempo y capacidad económica; es decir, "le da la opción al estudiante de iniciar su camino educativo e ir adelantando o dando pasos siempre hacia adelante con la certeza de que su recorrido lo conducirá a una formación más compleja en el saber y el hacer" (Fernández, Macías, Gómez, Chico, 2010, p. 33) y, por consiguiente, tener una mejor calidad de vida.

La formación por ciclos propedéuticos o niveles secuenciales es una respuesta a las exigencias del mercado laboral que requiere de personas con habilidades y destrezas que le permitan atender con pertinencia y certeza la evolución tecnológica, ya que la aplicación de buenas prácticas de las TIC permite retroalimentar y desarrollar la ciencia así como asegurar la productividad de todos los procesos.
Teniendo en cuenta que estas reflexiones se suscriben en el nivel de pregrado, el estudiante puede ingresar al nivel técnico profesional, seguir al nivel tecnológico y terminar en el nivel profesional universitario. Los propósitos de formación de cada uno de estos niveles son diferentes pero complementarios y el eje integrador debe estar dado por las asignaturas o cursos del área profesional, la formación en investigación como elemento intrínseco al proceso de enseñanza-aprendizaje y el desarrollo de actitudes basadas en principios y valores que humanicen el ejercicio laboral. Al respecto, "es necesario motivar al estudiante hacia una investigación básica para fomentarle el espíritu creativo y crítico que lo induzca a estar en permanente estado de inquietud para aprender a aprender" (Fernández G., M., 2014, p. 65).

El Técnico Profesional desarrolla competencias con énfasis en un mayor hacer (destrezas) fundamentado en un saber básico (habilidades). Las competencias en el Tecnólogo están fundamentadas en un equilibrio entre el saber y el hacer y las del Profesional Universitario, en un mayor saber. En la búsqueda de la formación integral del estudiante, estas habilidades y destrezas deben estar sustentadas en actitudes (ser) que contribuyan a humanizar el conocimiento y su aplicación.

Por lo tanto, el proceso de enseñanza-aprendizaje debe estar orientado al desarrollo de habilidades y destrezas en correspondencia con los propósitos de formación del nivel que cursa el estudiante y es el docente quien tiene esta gran responsabilidad, como agente determinante de la buena calidad de este proceso.

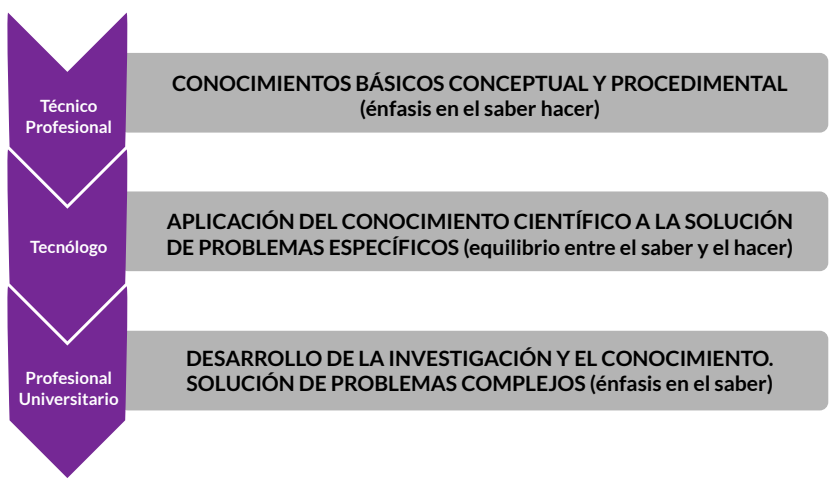

Figura 1. Alcances de la Formación Técnica Profesional, Tecnológica y Profesional Universitaria en Colombia. Fuente: Fernández, Marta M. (2007). 


\section{REQUERIMIENTOS DESDE LA EDUCACIÓN}

Se vive una época dinámica y fascinante que requiere de comunidades educadas y con un comportamiento responsable y de respeto por los demás lo que exige, como se viene exponiendo, de una educación bien administrada que forme personas que contribuyan no sólo a generar la satisfacción material sino, también, ambientes de tranquilidad, y para ello son necesarias las siguientes condiciones:

La educación, como factor de mayor incidencia en los cambios que se están dando en el mundo a través del tiempo; paradójicamente, los responsables directos de la misma, en efecto quienes orientan el proceso de enseñanza aprendizaje -docentes-, algunos de ellos, no responden con la misma dinámica evolutiva y hay momentos en que no perciben las nuevas característica de su entorno y presentan un comportamiento obsoleto en su quehacer académico.

En este comportamiento obsoleto inciden la indiferencia del Estado y de las directivas académicas de algunos países, toda vez que con ella no permiten la concreción y aplicación de programas relevantes que propicien el aseguramiento de la calidad de la educación, como los relacionados con la cualificación docente, así como programas que crean condiciones que le generen al profesor un entorno agradable, o sea un mejor vivir, para que las personas con mayor disposición hacia la ciencia y su aplicación aprecien la profesión de enseñar como una buena alternativa.

\section{Cultura educacional}

La educación debe ser prioridad para una sociedad y concebirse como un proceso perma- nente a través de la vida; debe ser vista como algo intrínseco en el quehacer de la persona. La educación superior es la que le da a la persona las competencias más acertadas para intervenir con creatividad y efectividad en el mercado laboral y generarle prosperidad socioeconómica. Cada etapa del proceso de formación tiene objetivos diferentes que deben estar armónicamente integrados para que sean útiles en esta era de la Economía del Conocimiento. Reflexionar sobre la enseñanza exige tener claridad sobre los agentes que intervienen directamente en el proceso de enseñanza aprendizaje -profesor - estudiante-, de aquellos que inciden en la generación del ambiente que propicie una buena enseñanza y un buen aprendizaje -padres de familia, instituciones educativas-, así como también las características geopolíticas y socioeconómicas del contexto.

\section{FORMACIÓN INTEGRAL DEL DOCENTE}

La formación integral de las personas le exige al docente tener también una formación integral para que oriente un aprendizaje responsable, entendiendo esta última como la armonización de su formación profesional (médico, economista...), con una pedagógica que les permita ejercer la enseñanza de manera consecuente con los propósitos de formación del estudiante, apoyándose en herramientas tecnológicas que faciliten la flexibilidad curricular, enmarcada en principios y valores ético

Esta formación integral posibilita al profesor para que sus habilidades, destrezas y actitudes adquiridas le permitan enfrentar y responder con creatividad y objetividad ante situaciones inesperadas propias de la relaciones profesorestudiante.

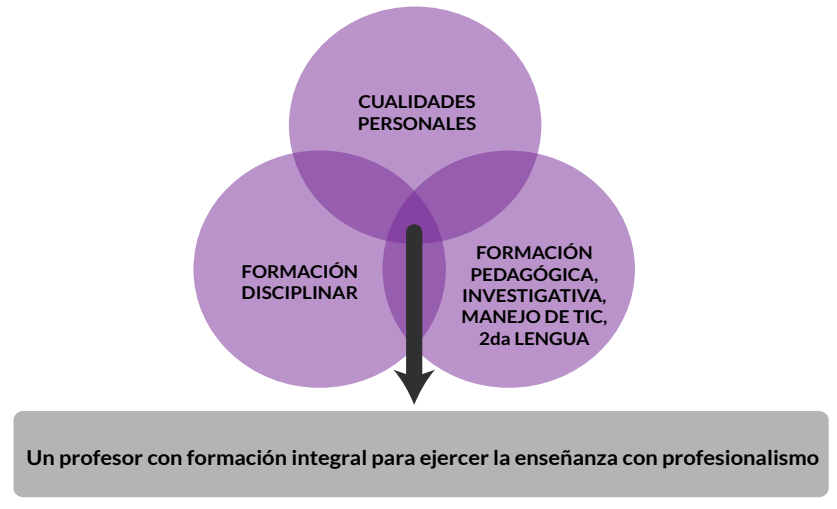

Figura 2. Formación Integral del Profesor.

Fuente: Fernández G., M. (2009). 


\section{Propuesta de enseñanza DIFERENCIADA E INTEGRADORA}

La enseñanza es la concreción y la concertación de las teorías y técnicas de la pedagogía. Debe ser vista y ejercida como una profesión, ya que enseñar es un arte y una ciencia, como lo han afirmado varios autores. Es arte, porque su práctica propicia en la persona sabiduría que le permite controlar sus instintos para lograr una vida digna, y es ciencia porque es una acción soportada por un conjunto de conocimientos, en permanente revisión y crecimiento, de un área específica del saber. Al respecto Woods, P. (1998) manifiesta que "decir que la enseñanza es una ciencia es afirmar que se trata de una actividad racional, sujeta a principios y leyes generales que la investigación puede descubrir" (p. 30).

Una enseñanza pertinente debe estar encaminada a la formación integral de las personas, esto es el equilibrio en el desarrollo de las capacidades cognitivas, motrices, comunicativas y afectivas que permitan conservar la especificidad cultural de cada región, utilizar los aspectos positivos de la economía de mercado, apreciar otras culturas y aprovechar los adelantos tecnológicos que proporcionan buenas condiciones en la praxis de vida. Dadas las tendencias contemporáneas hacia un aprender para la vida, los sistemas educativos están respondiendo a este propósito y, en el caso colombiano, la educación superior se ha estructurado por ciclos propedéuticos o secuenciales a nivel de pregrado: Técnico Profesional, Tecnológico y Profesional Universitario, y a nivel de postgrado: especialización, maestría y doctorado.

Esta estructuración permite la movilidad entre los diferentes niveles de formación, y entre éstos y el mundo laboral. Posibilita que un estudiante desarrolle las competencias laborales generales y específicas que le permiten el acceso a un trabajo; al mismo tiempo, le dan la opción de desarrollar competencias requeridas para continuar su formación en niveles superiores de educación; es decir, puede iniciar su camino educativo avanzando con la certeza de que su recorrido lo conducirá a una formación más compleja en el saber y hacer de acuerdo, como se ha venido sustentando, con su interés personal y capacidad económica.

Esta formación exige de una enseñanza pertinente y coherente con los propósitos del nivel académico que cursa el estudiante, es decir, una enseñanza diferenciada e Integradora que asegure el desarrollo de las competencias esperadas y responda a las exigencias de la Sociedad del Conocimiento la que requiere de personas en permanente formación para acrecentar el conocimiento y aplicarlo a lo largo de la vida, mediante un aprender a aprender.

En consecuencia, se propone que el docente ejerza la docencia basándose en una enseñanza diferenciada e Integradora, entendiéndola como aquella que le permite orientar y adecuar los métodos y herramientas pedagógicas de manera que el estudiante desarrolle sus capacidades en forma consecuente con los propósitos de formación del programa académico que cursa y le permita, así mismo, continuar su camino formativo aprovechado todo lo aprendido. Es un caminar siempre hacia adelante basado en un saber aprender.

La enseñanza diferenciada e integradora requiere que el docente tenga una previa y clara comprensión de los siguientes conceptos:

- Ciencia, tecnología y técnica.

- Alcances de formación: Técnica Profesional, Tecnológica y Profesional Universitaria.

- Propósitos de formación de los diferentes métodos o estrategias pedagógicas.

- Formación por competencias, Flexibilidad e Interna nacionalización curricular

- Métodos pedagógicos y sus propósitos

- Las TIC como herramientas pedagógicas.

\section{RefLEXIONES finAles}

Las singularidades expuestas en estas reflexiones, basadas en el ser humano como centro de toda actividad propia de la sociedad del conocimiento o de la economía del conocimiento que caracteriza a la aldea global, exige una enseñanza enfocada en la formación continua de las personas, concepción que está prevaleciendo como respuesta a la dinámica actual de la ciencia y la técnica.

La enseñanza diferenciada e integradora es una propuesta pertinente en el escenario que se vive ya que es coherente con los propósitos de una formación secuencial o por ciclos, que se concibe para responder a los niveles de pregrado de la educación superior en Colombia: Técnico Profesional, Tecnológico y Profesional Universitario. Consiste en una manera de orien- 
tar el aprendizaje hacia el desarrollo, por parte del estudiante, de las habilidades y destrezas de acuerdo con los propósitos del nivel de formación en que se encuentra matriculado.

Para su aplicación, es determinante la calidad del cuerpo docente y en ello inciden aspectos como la consistencia de su formación, las condiciones físicas, logísticas y laborales de la institución, así como las disposiciones del gobierno y las políticas de Estado que se expidan en la búsqueda de darle posicionamiento al ejercicio de la docencia como una profesión relevante para el desarrollo socioeconómico de los pueblos.

La sociedad del conocimiento o de la economía del conocimiento como se le viene llamando a nuestra a aldea global, presenta singularidades tales como las que se anotan a continuación (Fernández G., M. 2014, p. 26).

- El ser humano con su conocimiento es el eje de la vida.

- La ciencia, la técnica y la tecnología son relativas en el tiempo.

- El conocimiento se apropia, analiza y aplica con una visión internacional.

- La dinámica de la ciencia exige un saber actualizado de la misma para su óptima interpretación y aplicación

- El costo de la tecnología no permite improvisación en su aplicación.

- Las exigencias laborales competitivas requieren de una formación basada en un saber aprender.

- Los diferentes sectores que conforman una so- ciedad perciben la educación como la estrategia determinante del desarrollo económico, concebido éste como la integración del crecimiento económico y el progreso social.

- Aprender para la vida, es decir, la formación continua y armonizada, ya es apreciada como una necesidad prioritaria por una Nación, lo que le exige al Estado y a los gobiernos políticas claras y efectivas que respondan a esta nueva concepción de Aprender para la Vida.

- Necesidad de espacios de convivencia en las instituciones de educación en donde sus actores interactúen para reflexionar en forma activa y crítica sobre el conocimiento y la manera como éste se relaciona con los paradigmas vigentes en una sociedad.

- Exigencias de una cultura profesional entre los profesores, caracterizada por la disposición al cambio, el hábito de estudio y la colaboración como perspectiva educativa que les permita formular metas compartidas y les proporcione una mayor participación y autonomía.

- El docente debe ejercer la enseñanza con profesionalismo y esto sólo se logra queriendo y respetando la profesión de enseñar.

Los profesores extraordinarios son los que están al día de los desarrollos intelectuales, científicos o artísticos de importancia en sus campos, razonan de forma valiosa y original en sus asignaturas, estudian con cuidado y en abundancia lo que otras personas hacen en su disciplina, leen a menudo muchas cosas de otros campos (en ocasiones muy distantes del suyo propio) y ponen mucho interés en los asuntos generales de su disciplina (Bain, 2006, p. 26) 
Referencias bibliográficas

Andrade, E. (1994). El Papel de la Educación en Tecnología en el Desarrollo Nacional de Países del Tercer Mundo. Recuperado de http://www.geocities.com/Athens/8478/ANDRADE.htm.

Banco Mundial. (2002). Construir Sociedades de Conocimiento: Nuevos Retos para la educación terciaria. Recuperado de http://siteresources.worldbank.org/TERTIARYEDUCATION/Resources/Documents/Constructing-KnowledgeSocieties/CKS-spanish.pdf

Bain, K. (2006). Lo que hacen los mejores profesores universitarios. Madrid. España.

Borrero Cabal, A., S.J. (2001). Administración de la investigación en la universidad. Investigación unida a la docencia. Conferencia XXVI, Simposio Permanente sobre la Universidad. Bogotá, Colombia.

Brunner, J. J. (2000). Globalización y el futuro de la educación: tendencias, desafíos, estrategias. Seminario sobre prospectivas de la Educación en la Región de América Latina y el Caribe, organizado por la Oficina Regional de Educación de la UNESCO. Santiago de Chile, Chile.

Centro Interuniversitario de Desarrollo - CINDA (2012). Aseguramiento de la Calidad en Iberoamérica. Educación Superior. Recuperado de http://www.cinda.cl/wp-content/uploads/2014/o2/Aseguramiento-de-la-calidad-enIberoam\%C3\%A9rica-2012.pdf

Delors, J. (2001). La educación encierra un tesoro. Informe para la UNESCO de la Comisión Internacional sobre Educación para el Siglo XXI. Madrid, España: Ediciones UNESCO.

Fernández, G. M. (2014) (Ed.). Enseñanza Diferenciada e Integradora: Una forma de enseñar para la vida. Cartagena, Colombia: Ediciones TECNAR.

Fernández, G.M., Macías, M.E., Chico, M., \& Gómez, M. (2010) (Ed.). Formación del Profesor para una Enseñanza Diferenciada: Una Respuesta a las Nuevas Tendencias de la Educación Superior. Cartagena, Colombia: Ediciones TECNAR. (p.33)

Fernández, G. M. (2008). Propuesta de Formación Pedagógica del Profesorado de la Educación Superior. Fundación Tecnológica Antonio De Arévalo. (Tesis de doctorado) Universidad Complutense de Madrid, Madrid, España.

De Ferranti, D., Perry, G., Lederman, D., y Maloney, M. (2002). De los Recursos Naturales a la Economía del Conocimiento. Estudios sobre América Latina y el Caribe, Puntos de Vistas. Bando Mundial, Washington.

Gómez, B. H. (1999). Educación. La Agenda del Siglo XXI. Bogotá, Colombia: Tercer Mundo.

González, J. F. (2005) Investigar sobre la actividad educativa: trascendencia en la formación de los docentes. Revista Complutense de Educación. (16 - 2), p.715

González, F. \& Macías, M. E. (2004). En torno al tema de la calidad en educación. Realidad y leyes. Revista Complutense de Educación. $(15-1)$ p.303

Instituto Colombiano para el Fomento de la Educación Superior - ICFES. (2001) (Ed.). Guía para la internacionalización de las instituciones de educación superior de Colombia. Bogotá, Colombia: Procesos Editoriales.

Macías, E. (2001). Persona y diversidad. Implicaciones educativas en la sociedad del conocimiento. Revista Complutense de Educación. (12-2), p. 611.

Martínez, A. M. (enero de 2015) Agenda 2030 para el Desarrollo Sostenible. Transformando Nuestro Mundo. Recuperado http://quadernsanimacio.net/

Naciones Unidas- Asamblea General. Proyecto de documento final de la cumbre de las Naciones Unidas para la aprobación de la agenda para el desarrollo después de 2015. Recuperado de http://www.socialwatch.org/sites/default/files/Agenda2030-esp.pdf.

Saldaña, E. L. y Barragán, J. N. (2016). La Economía basada en el Conocimiento. Recuperado de http://www.ur.mx/ LinkClick.aspx?fileticket=GY8zmEJrUTY\%3D\&tabid=2636

Sánchez, C. y Ríos, H. (2011). La economía del conocimiento como base del crecimiento económico en México. Enl@ce Revista Venezolana de Información, Tecnología y Conocimiento, 8 (2), 43-6o. Recuperado de http://www.redalyc.org/ pdf/823/82319126004.pdf

Toffler, A. (1994). El Cambio de Poder. Barcelona, España: Plaza \& Janes S.A. 
Woods, P. (1998). Investigar el arte de la enseñanza. Barcelona, España: Editorial Paidós.

Tobón, S., Rial, A., Carretero, M. \& García, J. (2006) (Ed.). Competencias, Calidad y Educación Superior. Bogotá, Colombia: Cooperativa Editorial Magisterio. 
Revista incluida en los siguientes agregadores de contenidos
Revista incluida en los siguientes directorios

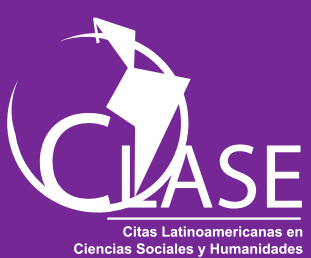

llboblat

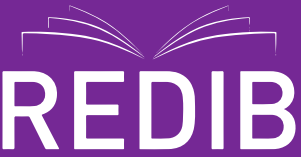

Red Iberoamericana

de Innovación y Conocimiento Cientifico

\section{Google Académico}
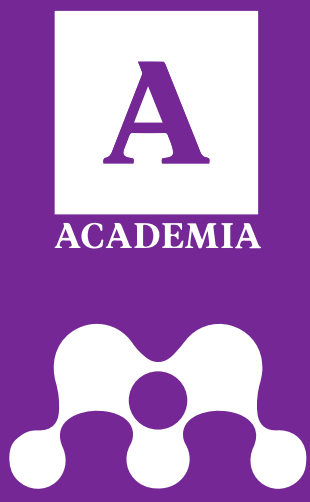

MENDELEY

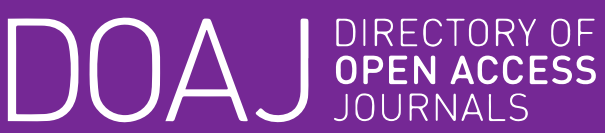

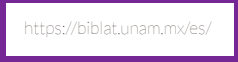
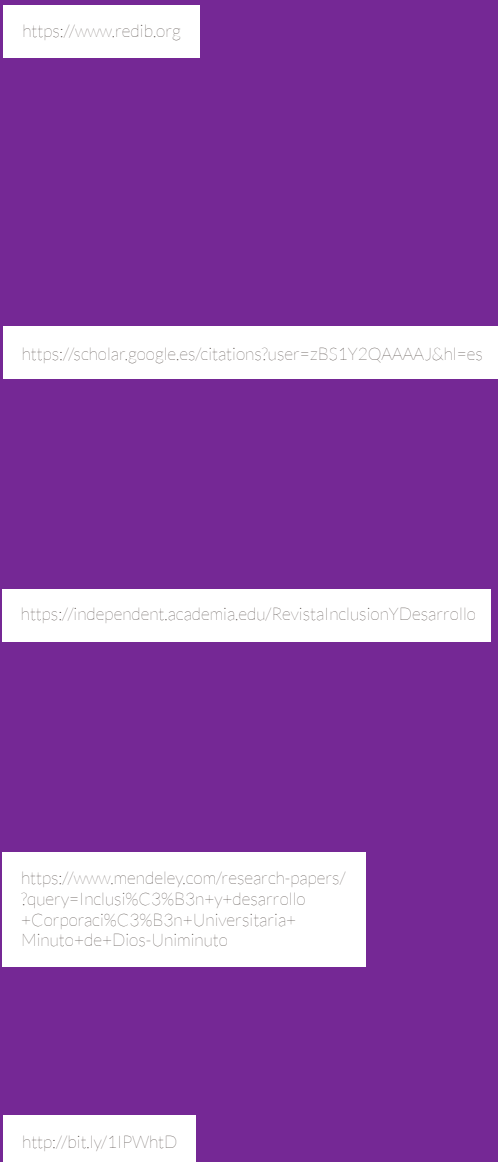

Revista incluida en la siguiente red social

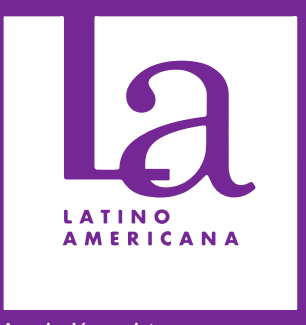



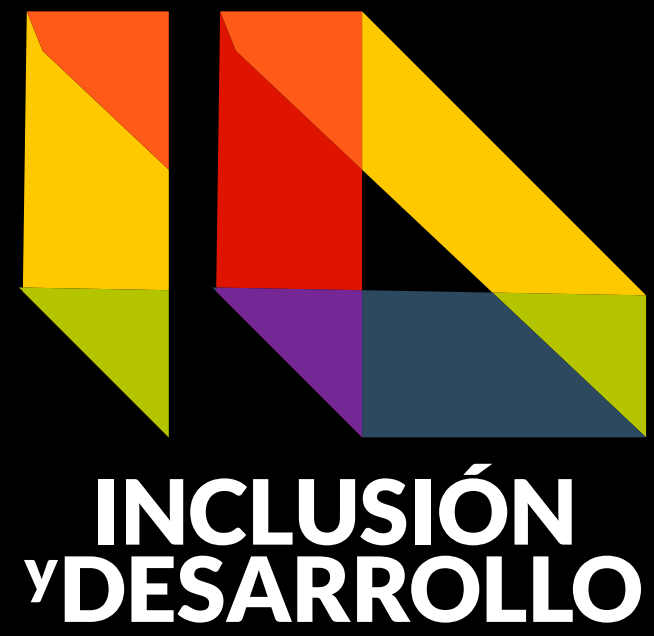

No. 1 Vol. 6 Año 2019 ISSN En línea: 2590-7700

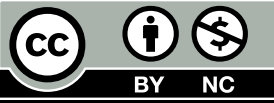

